\title{
Validación del Inventario de Fobia y Ansiedad Social - Forma Breve para universitarios argentinos
}

\author{
Valeria E. Morán ${ }^{1}$, Ana E. Azpilicueta, Marcos Cupani \\ Instituto de Investigaciones Psicológicas IIPsi, CONICET- UNC, Universidad Nacional de Córdoba, Argentina \\ Luis J. García- Lopez \\ Departamento de Psicología, División de Psicología Clínica, Universidad de Jaén, España
}

\section{RESUMEN}

Las problemáticas que afectan la capacidad de las personas para desempeñarse adecuadamente en las interacciones sociales, tales como el trastorno de ansiedad social (Social Anxiety Disorder, SAD), constituyen un ámbito de estudio que ha cobrado gran interés, debido a la importancia que las relaciones sociales tienen en todas las áreas de desempeño de los seres humanos. Sin embargo, se observa una escasez generalizada de estudios sobre ansiedad social en países latinoamericanos, en parte, debido a la ausencia de instrumentos construidos o adaptados a la población general o a conjuntos específicos. En función de ello, el objetivo del presente trabajo fue analizar las propiedades psicométricas del Inventario de Fobia y Ansiedad Social- Forma Breve (SPAI- B) para su utilización en estudiantes universitarios argentinos. Los resultados evidenciaron propiedades psicométricas adecuadas de validez y confiabilidad y se aportó evidencia de validez de grupos contrastados y convergente. Se discuten las limitaciones del estudio y nuevas líneas de investigación.

Palabras clave: Trastorno de ansiedad social (TAS); evaluación; estudiantes universitarios.

RESUMO - Validação do Inventário de Fobia e Ansiedade Social - Forma Breve para universitários argentinos

As problemáticas que afetam a capacidade das pessoas para terem um desempenho adequado nas interações sociais, como o transtorno de ansiedade social (Social Anxiet Disorder, SAD), constituem um âmbito de estudo que ganhou grande interesse devido à importância que as relações sociais têm em todas as áreas de desempenho dos seres humanos. No entanto, há um déficit generalizado de estudos sobre ansiedade social em países latino-americanos, devido a uma ausência de instrumentos construídos ou adaptados à população geral ou grupos específicos. Com base nisso, o objetivo do presente trabalho foi analisar as propriedades psicométricas do Inventario de Fobia e Ansiedade Social - Forma Breve (SPAI- B) para sua utilização com estudantes universitários argentinos. Os resultados mostraram propriedades psicométricas adequadas de validade e fidedignidade e gerou evidência de validade de grupos contrastados e validade convergente. As limitações do estudo e novas linhas de pesquisa são discutidas.

Palabras-chave: transtorno de ansiedade social (TAS); avalição; estudantes universitários.

\section{ABSTRACT - Validation of the Social Phobia and Anxiety Inventory - Brief Form for Argentinean college}

The problems that affect people's ability to perform adequately in social interactions, such as Social Anxiety Disorder (SAD), constitute an area of study that has gained great interest, due to the importance that social relations have in all areas of performance of human beings. However, there is a general scarcity of studies on social anxiety in Latin American countries, partly because of the absence of instruments built or adapted to the general population or specific groups. Due to this, the objective of the present investigation was to analyze the psychometric properties of the Spanish version of the Social Phobia and Anxiety Inventory-Short form (SPAI - B) to be used in Argentine college students. The results showed appropriate validity and reliability psychometric properties and it also has been provided evidence of contrasting groups and convergent validity. The limitations of the study and new lines of research are discussed.

Keywords: Social Anxiety Disorder (SAD); assessment; college students.

El trastorno de ansiedad social (TAS) es un fenómeno cuyo estudio se ha incrementado en las últimas décadas (Gómez-Ortíz, García \& Ruiz, 2016). Este crecimiento en parte se debe al impacto que tienen las dificultades para relacionarse en los diferentes ámbitos donde los individuos se desenvuelven cotidianamente para su desarrollo personal y profesional.

La American Psychiatric Association (APA, 2013) en el DSM-5 define al TAS como un trastorno caracterizado por un miedo excesivo y persistente a las situaciones de

${ }^{1}$ Endereço para correspondência: Facultad de Psicología - Universidad Nacional de Córdoba. Dir.: Enfermera Gordillo, s/n, Ciudad Universitaria, CP 5000, Córdoba, Argentina. E-mail: moranvaleria@gmail.com

Fuente de financiación: La presente investigación ha recibido financiamiento del Consejo Nacional de Investigaciones Científicas y Técnicas (CONICET) 
interacción social, sostenido por un temor a que resulten vergonzosas o humillantes, y a un concomitante rechazo social. Además, este manual indica que el miedo y la ansiedad ha de interferir significativamente con la rutina normal de la persona, en su funcionamiento académico o laboral, en sus relaciones sociales, o causar un malestar clínicamente significativo con deterioro en estas áreas.

La prevalencia de TAS en población adulta ha sido estudiada principalmente en Estados Unidos y Europa. Teniendo en cuenta estudios más actuales, de acuerdo con la APA (2013) en Estados Unidos, la prevalencia estimada anual es de aproximadamente el $7 \%$, y en Europa es del 2.3 \%. Otros estudios realizados en Europa tomando también los criterios del DSM IV, han encontrado tasas de prevalencia del 15.6 \% en Suecia (Furmark, Tillfors, Stattin, Ekselius, \& Fredrikson, 2000) y $7.3 \%$ en Francia (Pélissolo, André, Moutard-Martin, Wittchen \& Lépine, 2000). En el resto del mundo se han observado cifras menores (entre el $0.5 \%$ y $2 \%$ ). La mayoría de los meta-análisis sobre epidemiologia del TAS indican que el promedio de tasa de prevalencia se aproxima al $7 \%$ (Rodríguez, Caballo, García-López, Alcázar \& LópezGollonet, 2003), lo cual fue corroborado más tarde por Bandelow y Michaelis (2015). Sin embargo, un estudio reciente indica que la prevalencia es del $4 \%$ en todos los países y que las tasas de prevalencia son más altas en los países occidentales con mayores ingresos (Stein et al., 2017). Siguiendo esta estimación, Knappe, Sasagawa y Creswell (2015) en su revisión sobre estudios epidemiológicos encontraron que el TAS es el segundo trastorno más común de todos los incluidos en el DSM-5. Por otro lado, resumen que cerca del $13 \%$ de la población reconoce haber padecido alguno de los criterios diagnóstico en algún momento de su vida. En este punto es importante considerar que el número de adolescentes y jóvenes adultos que reportan los miedos sociales, pero que no son diagnosticados con fobia social, es mucho más grande y varía entre el 24\% y el 47\% según estudios realizados en diferentes países como Estados Unidos (Ruscio et al., 2008) y Alemania (Esaú, Conradt \& Petermann, 2000; Wittchen, Stein, \& Kessler, 1999).

Los estudios epidemiológicos y descriptivos de la ansiedad social se han extendido a la población de estudiantes universitarios, siendo considerados como uno de los grupos más vulnerables (Castellanos Cárdenas, Guarnizo Castillo \& Salamanca Camargo, 2011; Cejudo \& Fernández, 2015; Lipton, Weeks, Daruwala \& De Los Reyes, 2016). Strahan y Conger (1998) informaron que el 33\% del grupo reclutado en su estudio con estudiantes de psicología indicaban haber padecido síntomas de fobia social. Más tarde, Russell y Shaw (2009) en su estudio de prevalencia indicaron que el $10 \%$ de los estudiantes universitarios encuestados reportaron padecer ansiedad social moderada a severa. Estos resultados son coincidentes con estudios posteriores (Brook \& Willoughby, 2015, 2016; Pereira, Moreira, Chaló, Sancho, Varela \& Oliveira, 2016) los cuales también encontraron que la mayoría de los estudiantes universitarios han experimentado síntomas de ansiedad social en su transcurso por la vida académica.

Estos antecedentes refieren a estudios realizados en América del Norte y Europa, quedando Latinoamérica desprovista de cifras epidemiológicas representativas en universitarios. Solo se reporta un estudio en México donde García, Flores, Gutiérrez, Rojas y Agraz (2008) informan que en esta misma población la prevalencia de fobia social fue del 3.9\%. Los autores también indican que a pesar de que la ansiedad social parece ser un problema frecuente en todos los estudiantes universitarios mexicanos, sus resultados revelan que el miedo a la evaluación negativa y el grado de dependencia emocional, como subdimensiones evaluadas para este constructo, fueron mayores en las mujeres.

Esta escasez de estudios sobre ansiedad social en países latinoamericanos en parte es debido a la carencia de instrumentos construidos o adaptados a la población general, o a conjuntos específicos, que evalúen el constructo (Salazar, Caballo, Arias, \& Equipo de Investigación, C. I. S. O., 2016). Por el contrario, en el resto de los países que han profundizado en su conocimiento, se han desarrollado diversos dispositivos para su evaluación, siendo los cuestionarios de autoinforme el método más utilizado dada la naturaleza subjetiva de la ansiedad social (García-López, De Los Reyes, \& Salvador, 2015).

Uno de los instrumentos más utilizados para medir ansiedad social (Salazar et al., 2016) es el Social Phobia and Anxiety Inventory, SPAI (Turner, Beidel, Dancu \& Stanley, 1989). Esta escala está compuesta por 45 ítems que se dividen en dos subescalas obtenidas por análisis factorial confirmatorio denominadas Fobia Social y Agorafobia. Por otro lado, mediante análisis factorial exploratorio evidenciaron la existencia de cinco factores de segundo orden para la escala de Fobia Social, los cuales fueron denominados Miedo a las interacciones sociales individuales, Temor a las interacciones sociales grupales, Síntomas cognitivos y somáticos, Evitación social y Molestias asociadas con ser el centro de atención (Turner, Stanley, Beidel, \& Bond, 1989).

Tomando la subescala de Fobia Social, se creó el SPAI-B. Se trata de la versión breve española del SPAI creada para medir ansiedad social evitando las dificultades de la escala original debido a su excesiva longitud y despojándose del lenguaje heterocéntrico (García-López, Hidalgo, Beidel, Olivares \& Turner, 2008). Este instrumento ha sido validado para su aplicación en adolescentes y estudiantes universitarios de habla hispana (Piqueras, Espinosa-Fernández, García-López \& Beidel, 2012) obteniendo evidencias de confiabilidad y validez.

El SPAI-B está compuesto por 16 ítems que miden los aspectos somáticos, conductuales y cognitivos de la ansiedad social utilizando una escala tipo Likert de cinco puntos con la que la persona debe indicar la frecuencia con que experimenta diferentes síntomas. Los estudios 
psicométricos indican que permiten obtener una medida unidimensional equivalente a la versión completa multidimensaional del SPAI, tiene buena estabilidad temporal (García- López et al., 2008), es sensible al cambio terapéutico (García- López, Muela, Espinosa-Fernández \& Díaz-Castela, 2009; García- López, del Mar Díaz-Castela, Muela-Martínez \& Espinosa-Fernández, 2014) y tiene buen poder discriminativo (García-López, Sáez-Castillo, Beidel \& La Greca, 2015). Los estudios realizados con muestras universitarias (Piqueras et al., 2012) evidenciaron adecuada consistencia interna $(\alpha=.89)$ y correlaciones altas significativas con miedo a la evaluación negativa, y con afecto positivo y negativo. Por otro lado, también aportaron validez de grupos contrastados según el género, encontrando diferencias significativas a favor de las mujeres con tamaños del efecto pequeño. Estas diferencias han sido encontradas en diferentes estudios sobre ansiedad social utilizando diferentes medidas (Caballo, Arias, Salazar, Irurtia, \& Hofmann, 2015; Xu et al., 2012).

Con respecto a instrumentos válidos y confiables para ser empleados en la población argentina, recientemente se construyó el Test de Ansiedad Social (Morán, Olaz, Pérez \& Del Prette, 2018) específicamente para estudiantes universitarios. Si bien este instrumento posee adecuadas propiedades psicométricas, el mismo evalúa la intensidad con que los estudiantes experimentan ansiedad o nerviosismo ante situaciones específicas, dejando de lado la evaluación de los componentes somáticos y conductuales propios del TAS, por lo que contar con una medida complementaria que brinde esta información resulta de especial interés.

En este marco, el objetivo del presente trabajo fue analizar las propiedades psicométricas de la versión española del SPAI-B para la medición de la ansiedad social en estudiantes universitarios argentinos. Se espera encontrar una estructura unidimensional con índices de ajuste adecuados, y válida para hombres y mujeres, una consistencia interna satisfactoria, sensibilidad para detectar diferencias en el constructo entre grupos, y relaciones significativas entre las puntuaciones de la escala con puntajes de instrumentos semejantes

Contar con un instrumento de autoinforme de extensión breve supone ventajas en economía de tiempo a la hora de evaluar intervenciones y de obtener datos en contextos de investigación. En este punto, se torna relevante la aplicación en muestras universitarias teniendo en cuenta que se trata de una población que ha sido poco estudiada en Argentina y especialmente vulnerable con alta prevalencia de problemas de funcionamiento social (Morán et al., 2018).

\section{Método}

\section{Participantes}

La muestra estuvo constituida por 250 estudiantes universitarios (66 \% mujeres y $34 \%$ hombres) de
16 carreras pertenecientes a Universidades públicas y privadas de Córdoba y Rio Cuarto, Argentina. El rango de edad abarcaba de los 18 a los 30 años $(M=20.39$, $D E=1.94)$. El método de muestreo fue no probabilístico de tipo accidental. Teniendo en cuenta la naturaleza ordinal de los datos y la utilización de métodos robustos de estimación, se considera que el tamaño de la muestra fue adecuado (Muthen, du Toit, \& Spisic, 1997; Tabachnick \& Fidell, 2014; Tomarken \& Waller, 2005)

\section{Instrumentos}

Inventario de Fobia y Ansiedad Social- Forma

Breve (SPAI- B; García- López et al., 2008). Es un cuestionario que evalúa la sintomatología de fobia social en adolescentes y universitarios. El SPAI-B consta de 16 ítems con una escala de respuesta de tipo Likert de 5 puntos. Los encuestados tienen que responder a la frecuencia con la que ocurre la situación descrita en el apartado $(1=$ Nunca, $5=$ Siempre $)$. Mediante análisis factorial exploratorio y confirmatorio se evidenció una estructura unifactorial con una consistencia interna excelente $(\alpha=89)$ utilizando muestras universitarias españolas (Piqueras et al., 2012). Para este estudio se utilizó la versión española sin modificaciones ya que la simpleza de la terminología empleada y la ausencia de terminología cultural específica permitió corroborar la equivalencia de las expresiones idiomáticas en ambos países.

Test de Ansiedad Social para estudiantes universitarios (TAS-U; Morán et al., 2018). Este instrumento fue construido para estudiantes universitarios argentinos. Utilizando una escala de 1 a 10 ( 1 =Nada; $10=$ Mucho), la persona debe indicar la intensidad con que experimenta ansiedad, nerviosismo o malestar ante determinadas situaciones sociales. El instrumento se compone de 27 ítems agrupados en cuatro factores obtenidos mediante análisis factoriales de tipo exploratorio y confirmatorio, denominados Ansiedad ante situaciones sociales con personas conocidas, compuesto por 10 ítems $(\omega=.86)$, Ansiedad ante situaciones de desempeño académico o laboral compuesto por siete ítems $(\omega=.88)$, Ansiedad a ser observado por otros en situaciones generales, compuesto por seis ítems $(\omega=.77)$ y Ansiedad ante situaciones de abordaje afectivo o sexual, compuesta por cuatro ítems $(\omega=.81)$.

\section{Procedimiento}

Los participantes fueron informados acerca del propósito del estudio previo a la participación, y firmaron un formulario de consentimiento informado que describe el objetivo, la voluntariedad de su participación y la naturaleza confidencial de los datos. El presente estudio forma parte de un proyecto de tesis doctoral aprobado por un comité evaluador que ha considerado los aspectos éticos según la Declaración de Helsinski, así como la ley 25.326 de Protección de los Datos Personales. Los datos fueron recolectados en las Instituciones universitarias durante el 
periodo de cursado y la labor fue llevada a cabo por estudiantes de psicología que fueron entrenados para tal fin.

\section{Análisis de datos}

En primer lugar, se realizaron análisis preliminares de los datos de la base. Utilizando el software SPSS se evaluó el patrón de valores perdidos para evaluar si el mismo respondía a una distribución aleatoria (Tabachnick \& Fidell, 2014).

Posteriormente, se realizó un análisis factorial confirmatorio (AFC) con el fin de replicar la estructura original unidimensional. Para ello se utilizó el método de estimación basado en coeficiente mínimos cuadrados ponderados robusto (WLSMV) ya que se adecúa a medidas de tipo ordinal (escalas Likert, por ejemplo), no requiere hipótesis de distribución y proporciona estimaciones menos sesgadas (Brown, 2015; Ferrando \& Lorenzo-Seva, 2013; Freiberg Hoffmann, Stover, de la Iglesia \& Fernández Liporace, 2013). Para evaluar el ajuste de los modelos, se utilizó el Índice de Ajuste Comparativo (CFI), el Índice de Tucker-Lewis (TLI), el Error Cuadrático Medio de Aproximación (RMSEA) y la ponderada Media Cuadrática Residual (WRMR). Los valores entre .90 y .95 o superiores para el CFI y TLI son considerados como ajustes aceptables a excelentes, se esperan valores entre .05 y .08 para el RMSEA y valores menores a 1 para WRMR (Yu \& Muthen, 2002). Los análisis se realizaron utilizado el software Mplus.

Para analizar la consistencia interna de la escala se calculó el índice de fiabilidad compuesta $(\omega)$ considerando como aceptables valores superiores a .70 y satisfactorios valores superior a .80 (Hogan, 2004). Las diferencias de acuerdo con el género se analizaron mediante prueba $t$ para muestras independientes con análisis de tamaño del efecto propuesto por Cohen (1988) siendo pequeño cuando adopta valores entre 20 y .49 , mediano para valores entre .51 y .79 , y grande para valores mayores a .80 . Las relaciones del test con otras variables fueron analizadas mediante el coeficiente de correlación Pearson tomando los criterios del mismo autor (Cohen, 1988) para su interpretación siendo un tamaño del efecto pequeño cuando la correlación se encuentra por debajo de .10 , medio en torno a .30 y grande cuando es mayor que .50 .

\section{Resultados}

\section{Análisis de los casos perdidos}

El porcentaje de casos perdidos para toda la escala no superó el 5\% establecido (Schafer, 1999), y el mismo respondía a una distribución aleatoria, por lo tanto, se procedió a imputar los datos ausentes por el modo.

\section{Análisis de ítems}

En segundo lugar, se procedió a los análisis descriptivos de los datos. Se calcularon estadísticos descriptivos de los ítems y se analizó la frecuencia de respuestas encontrando que en todos los ítems fueron seleccionadas todas las opciones de respuesta. Con respecto a la distribución, como puede observarse en la Tabla 1 los valores de asimetría y curtosis fueron adecuados y excelentes según los criterios de George y Mallery (2011) para adecuarse a distribución de tipo normal.

Tabla 1

Media (M), Desviación Estándar (DE), Asimetría (A) y Curtosis (C)

\begin{tabular}{lcccc}
\hline & $\mathrm{M}$ & $\mathrm{DE}$ & $\mathrm{A}$ & $\mathrm{C}$ \\
\hline Item1 & 2.79 & 1.10 & .26 & -.48 \\
Item2 & 2.66 & 1.08 & .24 & -.62 \\
Item3 & 3.33 & 1.16 & -.19 & -.80 \\
Item4 & 1.72 & 0.98 & 1.52 & 1.97 \\
Item5 & 2.39 & 1.17 & .49 & -.60 \\
Item6 & 2.53 & 1.09 & .49 & -.30 \\
Item7 & 2.31 & 1.09 & .49 & -.50 \\
Item8 & 2.37 & 1.06 & .42 & -.58 \\
Item9 & 1.95 & 1.14 & 1.13 & .43 \\
Item10 & 2.50 & 1.08 & .33 & -.44 \\
Item11 & 3.05 & 1.25 & .04 & -.97 \\
Item12 & 1.85 & 1.06 & 1.19 & .76 \\
Item13 & 2.36 & 1.04 & .58 & -.15 \\
Item14 & 2.06 & 1.10 & .92 & .10 \\
Item15 & 2.32 & 0.79 & .53 & .02 \\
Item16 & 2.01 & 0.78 & .83 & .58 \\
Total & 38.21 & 9.49 & .42 & .26 \\
\hline
\end{tabular}




\section{Análisis factorial confirmatorio}

El modelo original de 16 ítems presentó índices de ajuste que si bien no alcanzaron los valores considerados óptimos fueron muy cercanos $(\mathrm{CFI}=.88$, $\mathrm{TLI}=.86$, RMSEA $=.09$, WRMR $=1.22$ ). Los coeficientes de regresión estandarizados del modelo final presentaron valores significativos de entre .38 y .73 (Ver Tabla 2). Tomando en consideración estos resultados se evaluaron los índices de modificación (IM), los cuales indicaron una mejora en el ajuste del modelo a partir de la correlación entre los errores de los ítems 1 y 3 . Como puede observarse en la Tabla 3, hubo una leve mejoría en los índices, pero aún no alcanzaron los valores esperados, por lo que se decidió introducir una nueva correlación entre los errores de los ítems 7 y 9, según lo indicado por los IM. El modelo nuevamente presentó una leve mejoría, que permitió que los índices CFI y RMSEA alcanzaran los valores óptimos (ver Tabla 3). Los índices TLI y WRMR fueron adecuados según los criterios de Moral (2006), pero teniendo en cuenta que no fueron los esperados, se decidió inspeccionar si los datos podrían responder a otro modelo de más de un factor a través de un análisis factorial exploratorio utilizando el método de extracción de mínimos cuadrados no ponderados (ULS). Para la determinación del número de factores se obtuvo que la solución unidimensional resultó adecuada tanto con el método de Análisis Paralelo (Timmerman \& Lorenzo-Seva, 2011) como el método de Hull (Lorenzo-Seva, Timmermann \& Kiers, 2011). En base a estos resultados y a que los índices de ajuste fueron aceptables y muy cercanos a lo esperado, se decidió conservar el modelo sin introducir nuevas modificaciones.

Tabla 2

Coeficientes de regresión estandarizados

\begin{tabular}{|c|c|}
\hline Item & $\beta$ \\
\hline Item1 & $.65^{* *}$ \\
\hline Item2 & $.64^{* *}$ \\
\hline Item3 & $.47^{* *}$ \\
\hline
\end{tabular}

Tabla 2 (continuación)

Coeficientes de regresión estandarizados

\begin{tabular}{|c|c|}
\hline Item & $\beta$ \\
\hline Item4 & $.56^{* *}$ \\
\hline Item5 & $.62^{* *}$ \\
\hline Item6 & $.62^{* *}$ \\
\hline Item7 & $.52^{* *}$ \\
\hline Item8 & $.73^{* *}$ \\
\hline Item 9 & $.43^{* *}$ \\
\hline Item10 & $.54^{* *}$ \\
\hline Item11 & $.47^{* *}$ \\
\hline Item12 & $.38^{* *}$ \\
\hline Item13 & $.58^{* *}$ \\
\hline Item14 & $.54^{* *}$ \\
\hline Item15 & $.57^{* *}$ \\
\hline Item16 & $.59^{* *}$ \\
\hline
\end{tabular}

${ }^{* *} p \leq .001$

Con respecto a la correlación entre errores de ítems indica que hay una posible relación entre la varianza no explicada de ambos reactivos. La alta correlación entre los residuos puede deberse, entre otras cosas, a la superposición de contenido en la redacción de los ítems, o a la influencia de ciertos factores tales como las características de la demanda planteada, la aquiescencia, o la deseabilidad social (Brown, 2015). En base a esto, especificar estas correlaciones permite la obtención de un modelo más parsimonioso sin impactar en la representación del constructo. Específicamente, en los ítems 1 y 3 se indaga acerca de situaciones en que la persona es el centro de atención ante una audiencia, aunque en el ítem 3 se le da especificidad al determinar la demanda de la situación. En los ítems 7 y 9 se indaga sobre situaciones en las que la persona debe hablar sobre sí mismo, pero en el ítem 9 se especifica sobre qué aspecto de sí mismo. A pesar del solapamiento en la información entre los ítems mencionados, teniendo en cuenta que cada uno aportó información adicional especifica se decidió conservarlos.

Tabla 3

Índices de Ajuste del Modelo Contrastado

\begin{tabular}{lccccccc}
\hline \multicolumn{1}{c}{ Método de estimación } & $\chi^{2}$ & $g l$ & $\chi^{2} / g l$ & CFI & TLI & RMSEA [IC 90\%] & WRMR \\
\hline Modelo original & $331.02^{* *}$ & 104 & 3.18 & .88 & .86 & $.09[.08-.10]$ & 1.22 \\
Error ítems 1-3 & $303^{* *}$ & 103 & 2.94 & .89 & .87 & $.09[.08-.10]$ & 1.16 \\
Error ítems 7-9 & $282.35^{* *}$ & 102 & 2.76 & .90 & .88 & $.08[.07-.09]$ & 1.12 \\
\hline
\end{tabular}

${ }^{* *} p \leq .001$

Para observar si la estructura factorial difiere según el sexo se realizó un análisis de invariancia para el modelo configural, el modelo métrico y el modelo escalar. Cabe mencionar que las pruebas de diferencias entre los valores de $\chi^{2}$ se realizaron utilizando la corrección de Santorra-Bentler (2010), debido a que resulta más apropiada cuando se utiliza WLSMV como estimador. Los tres modelos obtuvieron índices de ajuste satisfactorios 
(Tabla 4). Las diferencias de $\chi^{2}$ entre el modelo de base (configural) y el modelo métrico no fueron significativas. En el modelo escalar se encontraron diferencias significativas pero dado que la prueba de chi cuadrado es sensible al tamaño de la muestra, basado en el criterio de
Chen (2007) se indica que en el modelo escalar no hubo alteraciones significativas en el ajuste ya que no hubo descenso mayor a 0.01 del CFI ni incremento mayor a 0.015 en el RMSEA por lo que se determina que la escala es invariante para ambos sexos.

Tabla 4

Índices de ajuste en el análisis de invarianza factorial según sexo

\begin{tabular}{lccccccccc}
\hline & $\chi^{2}$ & $g l$ & CFI & TLI & RMSEA & WRMR & $\Delta \chi^{2}$ & $\Delta$ CFI & $\Delta$ RMSEA \\
\hline Configural & 371.41 & 205 & .91 & .89 & .08 & 1.31 & & & \\
Métrica & 340.46 & 219 & .93 & .93 & .07 & 1.34 & 6.69 & +0.02 & -0.01 \\
Escalar & 425.36 & 282 & .92 & .93 & .06 & 1.52 & $102.56^{* *}$ & -0.01 & -0.01 \\
\hline
\end{tabular}

Nota. $g l=$ grados de libertad; $\Delta \chi^{2}=$ Diferencia en Chi Cuadrado; $\Delta$ CFI=Diferencia en CFI. ${ }^{* *} p \leq .001$

\section{Consistencia Interna}

La consistencia interna de la escala fue evaluada mediante el cálculo de fiabilidad compuesta, obteniendo $\omega=.89$, valor considerado muy bueno por la literatura (Hogan, 2004).

\section{Diferencia de grupos según género}

Para evidenciar si la escala es sensible a las diferencias del constructo entre grupos, se llevó a cabo un análisis de grupos contrastados. Los resultados $(t=2.4, p \leq .01)$ indicaron que las mujeres $(M=39.33$, $\mathrm{DE}=8.95)$ presentaron puntuaciones significativamente más altas que los hombres $(M=35.93, D E=9.35)$, con un tamaño del efecto pequeño $(d=.38)$ similar a los resultados encontrados por los autores originales del test.

\section{Estudio de validez convergente}

Se analizaron las correlaciones entre los puntajes del SPAI-B con los obtenidos en el TAS-U. Como puede observarse en la tabla 5, se obtuvieron correlaciones significativas positivas medianas a grandes entre todos los constructos. El SPAI-B presentó relaciones más fuertes con las subescalas del TAS-U de Ansiedad social en situaciones académicas y Ansiedad social ante desconocidos y en lugares públicos.

Tabla 5

Coeficientes de correlación entre el SPAI-B y las subescalas del TAS-U

\begin{tabular}{lcccc}
\hline & 1 & 2 & 3 & 4 \\
\hline 1. SPAI-B & - & & & \\
2. TAS- Con &. $\mathbf{4 0 * *}$ & - & & \\
3. TAS- Ac & $.52^{* *}$ & $.40^{* *}$ & - & - \\
4. TAS- Des & $.48^{* *}$ & $.39^{* *}$ & $.51^{* *}$ & $.57^{* *}$ \\
5. TAS- AfecSex & $.41^{* *}$ & $.47^{* *}$ & $.48^{* *}$ & - \\
\hline
\end{tabular}

Nota. SPAI-B=Inventario de Fobia y Ansiedad Social - Forma Breve; TAS-Con=Ansiedad Social ante personas conocidas; TAS-Ac=Ansiedad Social en situaciones académicas; TAS-Des=Ansiedad Social ante personas desconocidas y lugares públicos; TAS- AfecSex=Ansiedad social en situaciones de abordaje afectivo sexual. ${ }^{* *} p \leq .001$

\section{Discusión}

Las problemáticas que afectan la capacidad de las personas para desempeñarse adecuadamente en las interacciones sociales constituyen un ámbito de estudio que en la actualidad ha cobrado gran interés. Esto se explica por la importancia que las relaciones sociales tienen en todas las áreas de desempeño de los seres humanos, siendo tanto las referidas a los vínculos como así también las profesionales y laborales.
El objetivo del presente trabajo fue realizar estudios de validación del Inventario de Fobia y Ansiedad SocialForma Breve (SPAI-B; García- López et al., 2008) para su utilización en estudiantes universitarios argentinos. Para ello se evaluó la estructura factorial de la escala, su consistencia interna, y se otorgó evidencia de validez de grupos contrastados y validez convergente.

Con respecto a la estructura interna, mediante análisis factorial confirmatorio se pudo constatar que la escala presenta una estructura unidimensional al igual que 
las versiones españolas para estudiantes universitarios (Piqueras et al., 2012) y adolescentes (García- López et al., 2008). Este factor único también fue evidenciado por Roberson-Nay, Strong, Nay, Beidel y Turner (2007) en su versión para adultos de habla inglesa y por Olivares, García-López, Hidalgo y Caballo (2004) con adolescentes. Este factor ha sido denominado por los diferentes autores como ansiedad social general contemplando los aspectos cognitivos, conductuales y somáticos característicos del fenómeno. Es importante tener en cuenta que estos modelos de única dimensión carecen de postulados teóricos consistentes que los sustenten, lo cual es una falencia recurrente en la medición de la ansiedad social. Puntualmente, Salazar et al. (2016) afirman que los instrumentos más utilizados a nivel internacional se han valido de procesos hipotético- deductivos para redactar e interpretar sus ítems (Fernández-Sogorb, del Pilar Aparicio-Flores, Granados, Aparisi-Sierra, \& Inglés, 2018) en base a criterios clínicos y establecer su estructura interna a partir de los resultados de análisis factoriales. No obstante, muchos de estos instrumentos cuentan con estudios que otorgan evidencia de otras fuentes de validez que permiten dar cuenta de la calidad de su medición.

En otro orden, referido a la consistencia interna del SPAI-B en la muestra estudiada, la escala obtuvo un coeficiente de frabilidad compuesta muy bueno (.89) según los criterios de Hogan (2004) lo que da cuenta de la precisión de la escala en sus medidas. Este valor es igual al obtenido en la validación en universitarios españoles (Piqueras et al., 2012) y similar a los hallados en otros estudios psicométricos llevados a cabo en otros países y lenguas, por ejemplo, la portuguesa (Vieira et al. 2013), tanto en la versión breve como en la versión completa del test.

En el análisis de diferencias según género se encontraron diferencias significativas con tamaño del efecto pequeño a favor de las mujeres. Estas diferencias no solo han sido encontradas en los estudios psicométricos de las diferentes versiones del SPAI (Clark et al., 1994; Piqueras et al., 2012) y de otros instrumentos para evaluar ansiedad social (Morán, 2017), sino también en distintos estudios epidemiológicos del fenómeno. Según el DSM-5, el trastorno de ansiedad social es más frecuente en las mujeres, y la diferencia entre géneros es más marcada en los adolescentes y los adultos jóvenes (APA, 2013). Otros estudios también revelan que las mujeres presentan mayores niveles de ansiedad social, sobre todo ante situaciones de evaluación social real (Caballo et al., 2015; Dell'Osso et al., 2015; MacKenzie \& Fowler, 2013; Stein et al., 2017).
Finalmente, el último estudio realizado demostró la versión argentina del SPAI-B presentan relaciones positivas con los puntajes del TAS-U aportando evidencia de validez convergente, teniendo en cuenta que este instrumento evalúa la intensidad con que estudiantes universitarios experimentan ansiedad social ante determinadas situaciones características de esta población, aunque sin discriminar los componentes conductuales, cognitivos y somáticos que son evaluados por el SPAI-B. La relación encontrada fue mayor en la dimensión de ansiedad social ante personas desconocidas en lugares públicos, en las cuales el contenido presenta situaciones en que es posible detectar el miedo a ser observado por otros, una de las características propias del Trastorno de Ansiedad Social.

A pesar de los satisfactorios resultados obtenidos, el siguiente trabajo presentó algunas limitaciones que indican la necesidad de continuar analizando las propiedades psicométricas de la escala para su utilización en población argentina. Principalmente, se utilizó un método de muestreo probabilístico lo cual reduce la probabilidad de representatividad de la muestra. No obstante, la factibilidad de aplicar este tipo de métodos de muestreo es reducida cuando se trabaja con este tipo de poblaciones por lo que se procuró incluir universidades públicas y privadas, diferentes disciplinas y alumnos de diversos niveles de cursado. Sumado a lo expuesto, es imprescindible ampliar los estudios de manera de poder obtener más evidencias de validez, por ejemplo, análisis de detección de cambios ante una intervención, estudios de invarianza estructural según género y comparación de poblaciones clínicas y sanas para el establecimiento de puntos de corte, tal como se ha realizado con muestras españolas (García-López, Beidel, Muela-Martínez \& EspinosaFernández, 2016).

Otro futuro estudio a realizar es la aplicación de otros modelos teóricos de la psicometría como el modelo de Rasch, que ha demostrado tener algunas ventajas con respecto a la Teoría Clásica de los Test. Por ejemplo, permite comparar los parámetros de las personas y de los ítems en un mismo continuo, así como establecer la invariancia de las medidas, entre otras virtudes (Cupani \& Cortez, 2016).

Teniendo en cuenta los resultados obtenidos en estos estudios, se puede concluir que el SPAI-B es un instrumento inicialmente valido y confiable para la medición de la ansiedad y la fobia social en estudiantes universitarios argentinos permitiendo economía de tiempo dado su breve extensión.

\section{Referencias}

American Psychiatric Association. (2013). Diagnostic and Statistical Manual of Mental Disorders (5th Edition). Arlington, VA: American Psychiatric Association. 
Bandelow, B., \& Michaelis, S. (2015). Epidemiology of anxiety disorders in the 21st century. Dialogues in clinical neuroscience, 17(3), 327-335.

Brook, C. A., \& Willoughby, T. (2015). The social ties that bind: Social anxiety and academic achievement across the university years. Journal of Youth and Adolescence, 44(5), 1139-1152. doi:10.1007/s10964-015-0262-8

Brook, C. A., \& Willoughby, T. (2016). Social anxiety and alcohol use across the university years: Adaptive and maladaptive groups. Developmental psychology, 52(5), 835. doi:10.1037/dev0000110.supp

Brown, T. A. (2015). Confirmatory Factor Analysis for Applied Research (2nd Ed.). New York: The Guilford Press.

Caballo, V. E., Arias, B., Salazar, I. C., Irurtia, M. J., \& Hofmann, S. G. (2015). Psychometric properties of an innovative self-report measure: The Social Anxiety Questionnaire for adults. Psychological assessment, 27(3), 997-1012.doi: 10.1037/a0038828

Castellanos Cárdenas, T.M., Guarnizo Castillo, C.A., \& Salamanca Camargo, Y. (2011). Relación entre niveles de ansiedad y estrategias de afrontamiento en practicantes de psicología de una universidad colombiana. International Journal of Psychological Research, 4(1), 50-57.

Cejudo, R., \& Fernández, A. (2015). Fobia social: Un fenómeno incapacitante. Revista Electrónica de Psicología Iztacala, $18(2)$, 836-851.

Chen, F. F. (2007). Sensitivity of goodness of fit indexes to lack of measurement invariance. Structural Equation Modeling: A Multidisciplinary Journal, 14(3), 464-504. doi: 10.1080/10705510701301834

Clark, D. B., Turner, S. M., Beidel, D. C., Donovan, J. E., Kirisci, L., \& Jacob, R. G. (1994). Reliability and validity of the Social Phobia and Anxiety Inventory for adolescents. Psychological Assessment, 6, 135-140. doi: 10.1037//1040-3590.6.2.135

Cohen, J. (1988). Statistical power analysis for the behavioral sciences (2nd ed.). Hillsdale, NJ: Erlbaum

Cupani, M., \& Cortez, F. D. (2016). Análisis psicométricos del Subtest de Razonamiento Numérico utilizando el Modelo de Rasch. Revista de psicología (Santiago), 25(2), 01-16. Recuperado de https://scielo.conicyt.cl/scielo.php?pid=S0719-05812016000200002\&script=sci_ arttext

Dell'Osso, L., Abelli, M., Pini, S., Carpita, B., Carlini, M., Mengali, F., ... \& Massimetti, G. (2015). The influence of gender on social anxiety spectrum symptoms in a sample of university students. Rivista di psichiatria, 50(6), 295-301.doi: 10.1708/2098.22688

Essau, C. A. Condrat, J. \& Petermann F. (2000). Frequency, comorbidity, and psychosocial impairment of anxiety disorder in German adolescents. Journal of Anxiety Disorders, 14(3), 263-278. doi:10.1016/s0887-6185(99)00039-0

Fernández-Sogorb, A., Aparicio Flores, P., Granados, L., Aparisi Sierra, D., \& Inglés, C. J. (2018). Ansiedad social y fobia social: Revisión de autoinformes y análisis de su fiabilidad y validez en muestra infantojuvenil española. Calidad de Vida y Salud, 11(1), 30-36. Recuperado de http://rua.ua.es/dspace/handle/10045/76046

Ferrando, P. J. \& Lorenzo-Seva, U. (2013). Unrestricted item factor analysis and some relations with item response theory. Technical Report. Department of Psychology, Universitat Rovirai Virgili, Tarragona. Recuperado de http://psico.fcep.urv.es/utilitats/factor/documentation/ technicalreport.pdf

Freiberg Hoffmann, A., Stover, J. B., de la Iglesia, G., \& Fernández Liporace, M. (2013). Correlaciones policóricas y tetracóricas en estudios factoriales exploratorios y confirmatorios. Ciencias Psicológicas, 7(2), 151-164. Recuperado de http://www.scielo.edu.uy/scielo.php?\%20 pid $=$ S1688-42212013000200005\&script $=$ sci_arttext\&tlng $=$ en

García, R. R., Flores, R. A. E., Gutiérrez, A. C. P., Rojas, M. A. Á., \& Agraz, F. P. (2008). Ansiedad social en estudiantes universitarios: Prevalencia y variables psicosociales relacionadas. Psicología Iberoamericana, 16(2), 54-62.

García-López, L. J., Beidel, D., Muela-Martínez, J. A., \& Espinosa-Fernández, L. (2016). Optimal cut-off score of Social Phobia and Anxiety Inventory-Brief form. European Journal of Psychological Assessment, 1-5. doi:10.1027/1015-5759/a000324

García-López, L. J., De Los Reyes, A., \& Salvador, M. C. (2015). Assessment of social anxiety in adolescents. En K. Ranta, A. M. La Greca, L. J. Garcia-Lopez, \& M. Marttunen (Eds.), Social anxiety and phobia in adolescents: Development, manifestation and intervention strategies (pp. 121-150). Cham, Switzerland: Springer International Publishing

García-López, L. J., del Mar Díaz-Castela, M., Muela-Martinez, J. A., \& Espinosa-Fernández, L. (2014). Can parent training for parents with high levels of expressed emotion have a positive effect on their child's social anxiety improvement? Journal of anxiety disorders, 28(8), 812-822.doi: 10.1016/j.janxdis.2014.09.001

García-López, L. J., Hidalgo, M. D., Beidel, D. C., Olivares, J., \& Turner, S. (2008). Brief form of the Social Phobia and Anxiety Inventory (SPAI-B) for adolescents. European Journal of Psychological Assessment, 24(3), 150-156.doi: 10.1027/1015-5759.24.3.150

García-López, L. J., Muela, J. M., Espinosa-Fernández, L., \& Diaz-Castela, M. (2009). Exploring the relevance of expressed emotion to the treatment of social anxiety disorder in adolescence. Journal of Adolescence, 32(6), 1371-1376. doi: 10.1016/j.adolescence.2009.08.001

García-López, L. J., Sáez-Castillo, A. J., Beidel, D., \& La Greca, A. M. (2015). Brief measures to screen for social anxiety in adolescents. Journal of Developmental \& Behavioral Pediatrics, 36(8), 562-568. doi: 10.1097/dbp.0000000000000213

George, D., \& Mallery, M. P. (2011). Using SPSS for Windows step by step: A simple guide and reference. Boston, MA: Allyn \& Bacon.

Gómez-Ortiz, O., Casas, C., \& Ortega-Ruiz, R. (2016). Ansiedad social en la adolescencia: Factores psicoevolutivos y de contexto familiar. Behavioral Psychology/Psicologia Conductual, 24(1), 29-50. Recuperado de https://www.redalyc.org/pdf/1339/133920328008.pdf

Furmark, T., Tillfors, M., Stattin, H., Ekselius, L., \& Fredrikson, M. (2000). Social phobia subtypes in the general population revealed by cluster analysis. Psychological Medicine, 30(6), 1335-1344.doi: 10.1017/s0033291799002615

Hogan, T. (2004). Pruebas Psicológicas: Una Introducción Práctica. México: Manual Moderno

Knappe, S., Sasagawa, S., \& Creswell, C. (2015). Developmental epidemiology of social anxiety and social phobia in adolescents. En K. Ranta, A. M. La Greca, L. J.Garcia-Lopez, \& M. Marttunen, Social Anxiety and Phobia in Adolescents (pp. 39-70). New York: Springer International Publishing.

Lipton, M. F., Weeks, J. W., Daruwala, S. E., \& De Los Reyes, A. (2016). Profiles of social anxiety and impulsivity among college students: A close examination of profile differences in externalizing behavior. Journal of Psychopathology and Behavioral Assessment, 38(3), 465-475. doi: 10.1007/s10862-015-9531-9

Lorenzo-Seva, U., Timmerman, M. E., \& Kiers, H. A. L. (2011). The Hull method for selecting the number of common factors. Multivariate Behavioral Research, 46(2), 340-364. doi:10.1080/00273171.2011.564527

MacKenzie, M. B., \& Fowler, K. F. (2013). Social anxiety disorder in the Canadian population: Exploring gender differences in sociodemographic profile. Journal of anxiety disorders, 27(4), 427-434. doi: 10.1016/j.janxdis.2013.05.006

Moral, J. (2006). Análisis factorial confirmatorio. En R. Landero \& M. T. González (Ed.), Estadística con SPSS y Metodología de la investigación (pp. 445-528). México: Trillas. 
Morán, V. E., Olaz, F. O., Pérez, E. R., \& Del Prette, Z. A. (2018). Desarrollo y validación del Test de Ansiedad Social para estudiantes universitarios (TAS-U). Liberabit, 24(2), 195-212.doi: 10.24265/liberabit.2018.v24n2.03

Muthén, B. O., du Toit, S. H. C., \& Spisic, D. (1997). Robust inference using weighted least squares and quadratic estimating equations in latent variable modeling with categorical and continuous outcomes. Retrieved from www.gseis.ucla.edu/faculty/muthen/articles/ Article_075.pdf

Olivares, J., Garcia-Lopez, L. J., Hidalgo, M. D., \& Caballo, V. (2004). Relationships among social anxiety measures and their invariance. European Journal of Psychological Assessment, 20(3), 172-179.doi: 10.1027/1015-5759.20.3.172

Pelissolo, A., André, C., Moutard-Martin, F., Wittchen, H. U., \& Lépine, J. P. (2000). Social phobia in the community: Relationship between diagnostic threshold and prevalence. European Psychiatry, 15(1), 25-28.doi: 10.1016/s0924-9338(00)00214-5

Pereira, A. S., Moreira, A. A., Chaló, P., Sancho, L., Varela, A., \& Oliveira, C. (2016). Development challenges of a full integrated app in higher education. Gaming and Technology Addiction, 117-139. doi:10.4018/978-1-5225-0778-9.ch007

Piqueras, J. A., Espinosa-Fernández, L., Garcia-Lopez, L. J., \& Beidel, D. C. (2012). Validación del Inventario de Ansiedad y Fobia socialForma Breve"(SPAI-B) en jóvenes adultos españoles. Behavioral Psychology/Psicología Conductual, 20(3), 505-529.

Roberson-Nay, R., Strong, D. R., Nay, W. T., Beidel, D. C., \& Turner, S. M. (2007). Development of an abbreviated Social Phobia and Anxiety Inventory (SPAI) using item response theory: the SPAI-23. Psychological Assessment, 19, 133-145.doi: 10.1037/1040-3590.19.1.133

Rodríguez, J. O., Caballo, V. E., Garcia-Lopez, L. J., Alcázar, A. I. R., \& Lopez-Gollonet, C. (2003). Una revisión de los estudios epidemiológicos sobre fobia social en población infantil, adolescente y adulta. Psicología Conductual, 11(3), 405-427.

Ruscio, A. M., Brown, T. A., Chiu, W. T., Sareen, J., Stein, M. B., \& Kessler, R. C. (2008). Social fears and social phobia in the USA: Results from the National Comorbidity Survey Replication. Psychological medicine, 38(1), 15-28.doi: 10.1017/S0033291707001699

Russell, G., \& Shaw, S. (2009). A study to investigate the prevalence of social anxiety in a sample of higher education students in the United Kingdom. Journal of Mental Health, 18(3), 198-206. doi:10.1080/09638230802522494

Salazar, I. C., Caballo, V. E., Arias, B., \& Equipo de Investigación, C. I. S. O. (2016). Validez de constructo y fiabilidad del Cuestionario de ansiedad social para adultos (CASO) en Colombia. Revista Latinoamericana de Psicología, 48(2), 98-107.doi: 10.1016/j.rlp.2015.07.001

Satorra, A., \& Bentler, P. M. (2010). Ensuring positiveness of the scaled difference chi-square test statistic. Psychometrika, 75(2), 243-248.doi: 10.1007/s11336-009-9135-y

Schafer, J. L. (1999). Multiple imputation: A primer. Statistical methods in medical research, 8(1), 3-15. doi: 10.1177/096228029900800102

Stein, D. J., Lim, C. C., Roest, A. M., de Jonge, P., Aguilar-Gaxiola, S., Al-Hamzawi, A., ... \& de Girolamo, G. (2017). The cross-national epidemiology of social anxiety disorder: Data from the World Mental Health Survey Initiative. BMC medicine, 15(1), 143.doi: 10.1186/ s12916-017-0889-2

Strahan, E., \& Conger, A. J. (1998). Social anxiety and its effects on performance and perception. Journal of Anxiety Disorders, 12, $293-305$. doi: 10.1016/s0887-6185(98)00016-4

Tabachnick, B. L., \& Fidell, L. S. (2014). Using multivariate statistics. (6th Ed.). Boston, MA: Pearson Education, Inc.

Timmerman, M. E., \& Lorenzo-Seva, U. (2011). Dimensionality assessment of ordered polytomous items with parallel analysis. Psychological Methods, 16(2), 209-220. doi:10.1037/a0023353

Tomarken, A. J., \& Waller, N. G. (2005). Structural equation modeling: Strengths, limitations, and misconceptions. Annual Review of Clinical Psychology, 1(1), 31-65. doi:10.1146/annurev.clinpsy.1.102803.144239

Turner, S. M., Beidel, D. C., Dancu, C. V., \& Stanley, M. A. (1989). An empirically derived inventory to measure social fears and anxiety. Psychological Assessment, 1(1), 35-40.doi: 10.1037//1040-3590.1.1.35

Turner, S. M., Stanley, M. A., Beidel, D. C., \& Bond, L. (1989). The social phobia and anxiety inventory: Construct validity. Journal of Psychopathology and Behavioral Assessment, 11(3), 221-234.doi: 10.1007/bf00960494

Vieira, S., Salvador, C., Matos, A. P., García- López, L. J., \& Beidel, C. (2013). Inventario de Fobia y Ansiedad Social-Version Breve: Propiedades psicometricas en una muestra de adolescentes portugueses. Behavioral Psychology/Psicologia Conductual, 21(1), 25-39.

Wittchen, H. U., Stein, M. B., \& Kessler, R. C. (1999). Social fears and social phobia in a community sample of adolescents and young adults: Prevalence, risk factors and comorbidity. Psychological Medicine, 29, 309-323. doi:10.1017/S0033291798008174

Xu, Y., Schneier, F., Heimberg, R. G., Princisvalle, K., Liebowitz, M. R., Wang, S., \& Blanco, C. (2012). Gender differences in social anxiety disorder: Results from the national epidemiologic sample on alcohol and related conditions. Journal of anxiety disorders, 26(1), 12-19. doi: 10.1016/j.janxdis.2011.08.006

Yu, C. Y., \& Muthén, B. (2002). Evaluation of model fit indices for latent variable models with categorical and continuous outcomes. Paper presented at the annual meeting of the American Educational Research Association, New Orleans, LA.

\section{Sobre os autores}

Valeria E. Morán é Profesora Doctora en Psicología. Becaria Posdoctoral CONICET. Especialista en Psicometría y estudios sobre comportamiento interpersonal.

Ana E. Azpilicueta é Lic. en Psicología. Becaria Doctoral CONICET.

Marcos Cupani é Profesor Doctor en Psicología. Investigador adjunto CONICET. Especialista en Psicométria y estudios sobre personalidad.

Luis J. García- Lopez é Profesor Doctor en Psicología. Especialista en estudios sobre Ansiedad Social. 